\title{
Environmental Color and the Cooperative Behavior of Children with Sensory Processing Challenges: An Exploratory Study
}

\author{
Marilyn Read \\ Oregon State University, Corvallis, USA \\ Email:marilyn.read@oregonstate.edu
}

How to cite this paper: Read, M. (2019). Environmental Color and the Cooperative Behavior of Children with Sensory Processing Challenges: An Exploratory Study. Creative Education, 10, 2448-2456.

https://doi.org/10.4236/ce.2019.1011173

Received: September 29, 2019

Accepted: November 15, 2019

Published: November 18, 2019

Copyright (อ 2019 by author(s) and Scientific Research Publishing Inc. This work is licensed under the Creative Commons Attribution International License (CC BY 4.0).

http://creativecommons.org/licenses/by/4.0/

Open Access

\begin{abstract}
The difficult challenge faced by occupational therapists and preschool teachers today is creating environments that benefit concurrently the behaviors of all children within one classroom setting. Occupational therapists and teachers design both the physical environment and the social environment for children with developmental needs across a broad spectrum of abilities. Children who have atypical reactions to sensory stimuli may benefit from a space with environmental color because it could act as a modifier for their attention in the environment. The purpose of this study was to investigate the impact of environmental color on the cooperative behavior of preschool children assessed with having one or more sensory processing challenges. It was predicted that walls with environmental color from focal colors (purple, blue, green, yellow, orange, and red) would positively impact the overall cooperative behavior of children with sensory processing challenges relative to a wall of white color scheme. The male participant showed higher cooperation scores in all visible-spectrum focal color conditions compared to the white color conditions. The cooperation scores for the female participant were lower on all visible-spectrum focal color conditions, except for orange, as compared with the white conditions. The results of the two different case studies within the environmental conditions are illustrated with discussion and implications of the research findings.
\end{abstract}

\section{Keywords}

Environmental Color, Preschool Children, Sensory Processing

\section{Introduction}

The designed environment of a preschool setting is a rich place of sensory sti- 
mulation for young children. Children's visual, tactile, auditory, gustatory, and olfactory senses are introduced to multiple stimuli in a preschool setting that can be inspiring or overwhelming for young children's experiences in the environment because they have different reactions to sensory stimuli. Children with sensory processing challenges can have a difficult time maneuvering through the stimuli in the preschool environment because the messages they receive are overwhelming to their central nervous systems (Kranowitz, 2003). The behavior of young children with sensory processing challenges is greatly impacted by the design of the classroom environment (Lawson \& Dunn, 2008). Children with extreme reactions to sensory stimuli may benefit from a space with environmental color because it may act as a modifier for their attention in the environment. Balancing the designed elements that promote children's success in the preschool environment with the space needs of children with a variety of developmental needs is the overall goal of an optimal preschool setting.

One design element that may be both useful to occupational therapists and teachers and effective in the designed preschool environment is color. The application of color on walls or floors has the potential to enhance the visual environment. When color is thoughtfully incorporated into a preschool setting, it is a visual stimulus that affords children a focal point of visual imagery. The perceptual dimensions of color are hue, brightness, and saturation (Bornstein, 2016) provide an opportunity to create a varied environment to young children. Gibson's (1979) theory provides researchers with a framework for studying color in the visual perceptual environment.

Based on Gibson's theory (1979), the Ecological Approach to Visual Perception, information provided by the environment to a child is abundant and complex with an emphasis on the visual features in the physical environment. Perception, learning, and behavior of children are impacted by the affordances in the environment. The child who learns through an affordance in an environment is discovering meaning according to Gibson (1979), and that meaning is perceived in that context (Gibson, 2000). Kyttä (2004) described the opportunity for researchers to consider affordances in the social context. The perception of the affordance influences the individual in ways that warrant empirical investigation.

Jasmin et al. (2018) synthesized empirical studies focusing on occupational therapy in preschools. They summarized that children had positive socio-emotional skills outcomes in occupational therapy when therapists utilized a multisensory approach to learning. Clearly, consideration of the senses is important for supporting children's success in the environment. Therefore, a wall with a visible-spectrum focal color may afford young children with sensory processing challenges a modifier as a background for play.

\section{Background}

This section reviews the empirical literature on color and human behavior; and 
the design of settings. Bornstein (2007) posited the importance of a basic hue categorization approach that includes red, yellow, green, and blue. This categorization is based on multiple studies that include psychophysical and perceptual approaches to empirical studies of infants and adults. Bornstein (2016) summarized the research on hue sensations of color as separate sensations. They include red and green; and yellow and blue. Focal colors are identified consistently as unique hues. It should be noted that this model focuses on color sensation. Pitchford and Mullen (2005) investigated the impact of perceived color categorization on preschool children's development of "conceptual colour space" (Pitchford \& Mullen, 2005: p. 70). They found that children were better at understanding focus colors when they looked at distant rather than adjacent color categories. Pitchford et al. (2009) found that participants of all ages, preschool elementary age, and adults preferred saturated colors to desaturated colors. Eliot (2015) proposed the need to develop sound theoretical work in the area of color and psychological functioning. The area of research is relatively new with room for empirical study and theoretical development.

Farran et al. (2012) studied the effects of color as an environmental cue in a maze activity. These researchers looked at participants that included adults with Williams Syndrome, typically developing adults, and typically developing children. They focused on comparing the results of mazes with focal colors and non-focal colors. Focal colors were those that were easy for young children to comprehend. Non-focal colors were created by averaging Red, Green, and Blue values across the spectrum. Results showed that for all participants the focal color condition showed fewer errors than the non-focal color condition. The colors that were easy for children to comprehend resulted in fewer errors when completing the maze activity. Focal color may positively impact children's wayfinding abilities.

Hidayetoglu et al. (2012) investigated color, lighting and wayfinding. They found that participants preferred warm colors and bright lighting levels. However, cool colors on the walls impacted the navigability abilities of participants in a positive direction in comparison to warm colors. Semantic differential items included: clear/blurry, attractive/unattractive, navigable, unnavigable, and inviting/repellent. For all four items, the neutral color was perceived as negative when compared with the warm color or the cool color.

Interventions using color stimuli have focused on children's handwriting performance and children's reading abilities. Imhof (2004) found that ADHD children showed improved attention and handwriting control when using colored paper over white paper. Children who chose to write on colored paper showed fewer poorly marked letters. The colors of the papers ranged from pastel to fluorescent colors. Iovino et al. (1998) found that blue colored overlays improved reading comprehension performance of all children as compared with red overlays and no overlays. Blue overlays reduced reading rates for all participants. Researchers in this study used blue overlays, red overlays, and no over- 
lays. Banaschewski et al. (2006) found that children with ADHD perceived color on the red-green axis more accurately than color on the blue-yellow axis. The researchers proposed that the cones in the eye that are receptive to short wavelengths in the visible spectrum may be more vulnerable than the medium and long wavelength cones.

Jalil et al. (2012) examined the impact that environmental color has on human behavior with a recommendation that investigators utilize actual settings and actual scenarios for the study of color in order to develop a clearer understanding of the impact of color on human behavior. Barrett et al. (2015) found in a multi-level analysis of design elements in classrooms that colors with high saturation and brightness were optimal when used as accents in a space rather than on an entire surface wall or floor. Joh and Spivey (2012) concluded that young children succeeded when using color as perceptual cues to solve a problem that focused on spatial reasoning. The colors used in the study were red, yellow, and blue. Dijkstra et al. (2008) investigated the role of color in the context of hospital rooms. They found that orange was effective for increasing arousal in participants who scored low on their ability to screen the stimuli around them. For this group of participants, green also had a stress-reducing effect when compared with white.

If colored overlays, color simulations, and colored paper have positive impacts on wayfinding, attention, and reading for participants, then it seems reasonable to propose that an environmental wall with color could positively impact the behavior of children with sensory processing challenges. It is predicted that they would be receiving varied environmental stimuli which would then help them to focus on the people and materials in front of the wall. A study by Read et al. (1999) found that children were more cooperative in a setting with a red wall as compared with the setting with a white wall. Therefore, children with sensory processing challenges may benefit in a similar way when viewing background color in the environment. The central hypothesis of this study is that background environmental wall color within the preschool classroom will positively impact the cooperative behavior outcomes of children with sensory processing challenges.

\section{Method}

A single subject research design was used to assess the impact of environmental color on the cooperative behavior of children with sensory processing challenges. Zhan and Ottenbacher (2001) discussed the value of single subject research designs for individuals with disabilities.

\subsection{Sample}

Following parental consent and children's assent via Institutional Review Board protocols, participants at an inclusive child development center were evaluated by the Early Childhood Education Coordinator utilizing the Sensory Profile Ca- 
regiver Questionnaire (Dunn, 2006). Scores of nine children were in the range of having sensory processing challenges. Eight additional children participated in the study. 17 children participated in the study ranging in age from 3 - 5 years. Data were analyzed for the two children who completed 12 video-recording sessions for the study.

\subsection{Instruments}

The Early Childhood Coordinator at the inclusive child development center screened children using the Sensory Profile Caregiver Questionnaire (Dunn, 2006). Children were evaluated on auditory processing, visual processing, vestibular processing, modulation related to body position and movement, modulation of movement affecting activity level, modulation of sensory input affecting emotional responses, modulation of visual input affecting emotional responses and activity level, and behavior and emotional responses. Participants were evaluated for color vision deficiency (i.e. color blindness) using a series of Standard Pseudoisochromatic Plates (Ichikawa, Hukami, Tanabe, \& Kawakami, 1978). The 17 participants did not have color vision deficiency.

\subsection{Procedure}

Children, randomly assigned to groups of 4 , participated in a clay-building activity in a space with white walls and full-spectrum fluorescent lighting for 10 minutes for each condition. Lawson and Dunn (2008) posited the importance of play preferences for children with sensory processing challenges. They found that children preferred play dough over other toys (Lawson \& Dunn, 2008). The lower section of the wall was covered with fabric up to 42 inches above the finished floor. A video-recording camera recorded children's behavior in the treatment condition for later data coding. The focus child sat facing the color or white wall in each condition. Each wall-color condition was video-recorded. Conditions were randomized with the control of a white background. For example: white, purple, white, green, white, blue, white, red, white, yellow, white, orange. Subsequently, participants were scored using a standardized measure of cooperative behavior, the Oregon Preschool Test of Interpersonal Cooperation (OPTIC) (Paulson, 1972). The OPTIC is a scale with 1) cooperation, 2) active interaction, 3) parallel play, 4) watching, 5) minimal interaction, and 6) obstructive interaction. Scores ranges from 5 points to 0 points. Three coders coded the video-recordings utilizing the OPTIC scale. Interrater reliability was $81 \%$. Two children (one boy and one girl) participated in all 12 conditions.

\section{Results}

Matthew, a pseudonym, was a 4-year-old boy who eagerly participated in the research activities. For all scores, his ratings on cooperative behavior were higher for the visible-spectrum focal color conditions as compared with the white conditions. The focal color scores were: Orange-3.7, Green-3.3, Purple-3.3, Blue-3.4, 
Red-3.5, and Yellow 3.1. For the white conditions the scores ranged from 2.8 3.2. See Figure 1. Matthew's scores support the study hypothesis.

Caroline, a pseudonym, was a 4-year-old girl who also readily participated in the research project. Caroline's scores do not support the study hypothesis. Except for the visible spectrum focal color orange, Caroline's cooperative behavior scores were lower on all visible spectrum focal color conditions as compared with the white conditions. The focal color scores were: Orange-3.8, Green3.1, Purple-3.2, Blue-3.0, Red-3.5, and Yellow 2.9. For the white conditions the scores ranged from 2.9 - 3.6. See Figure 2. Caroline's scores do not support the study hypothesis.

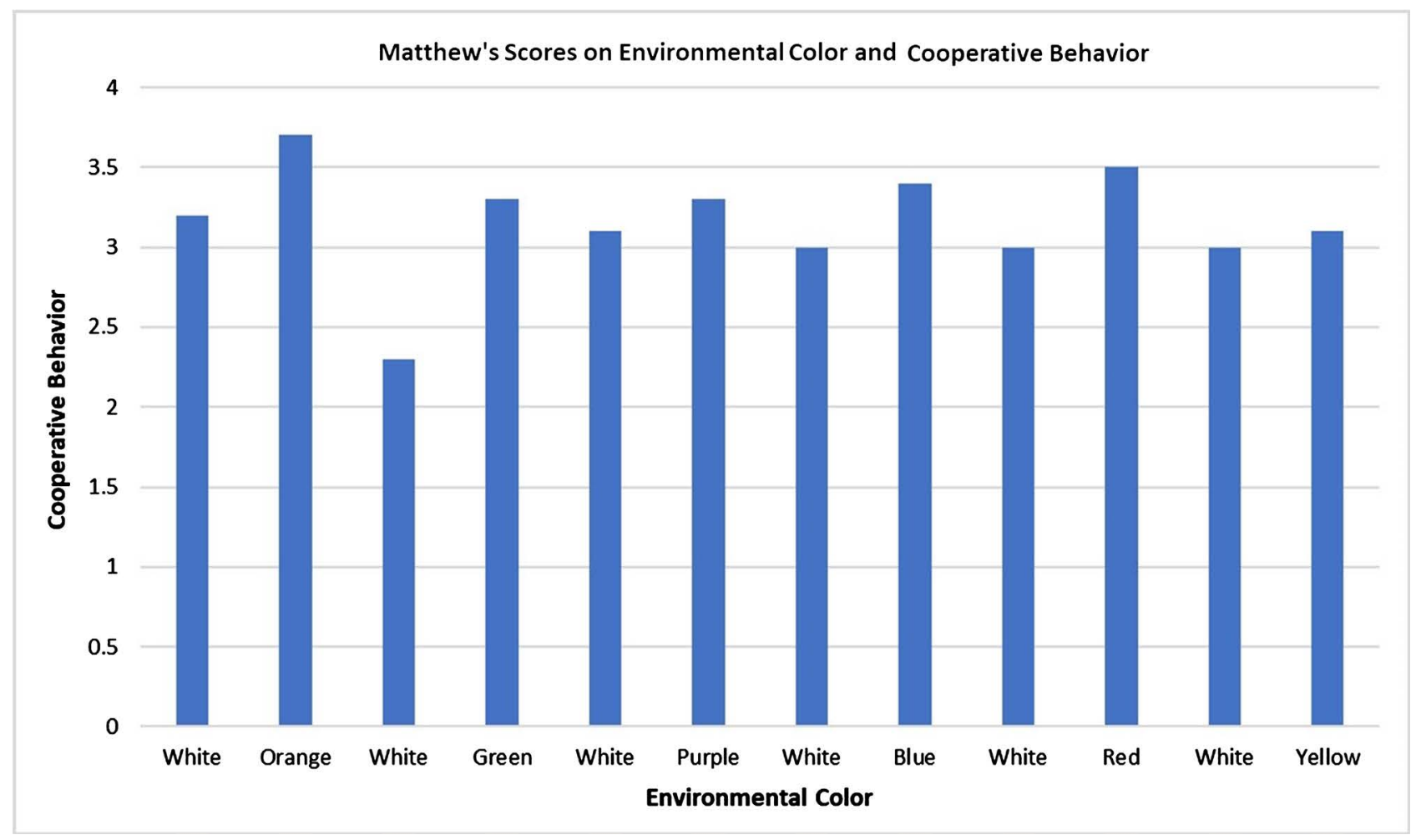

Figure 1. Matthew's scores on cooperative behavior and environmental color conditions.

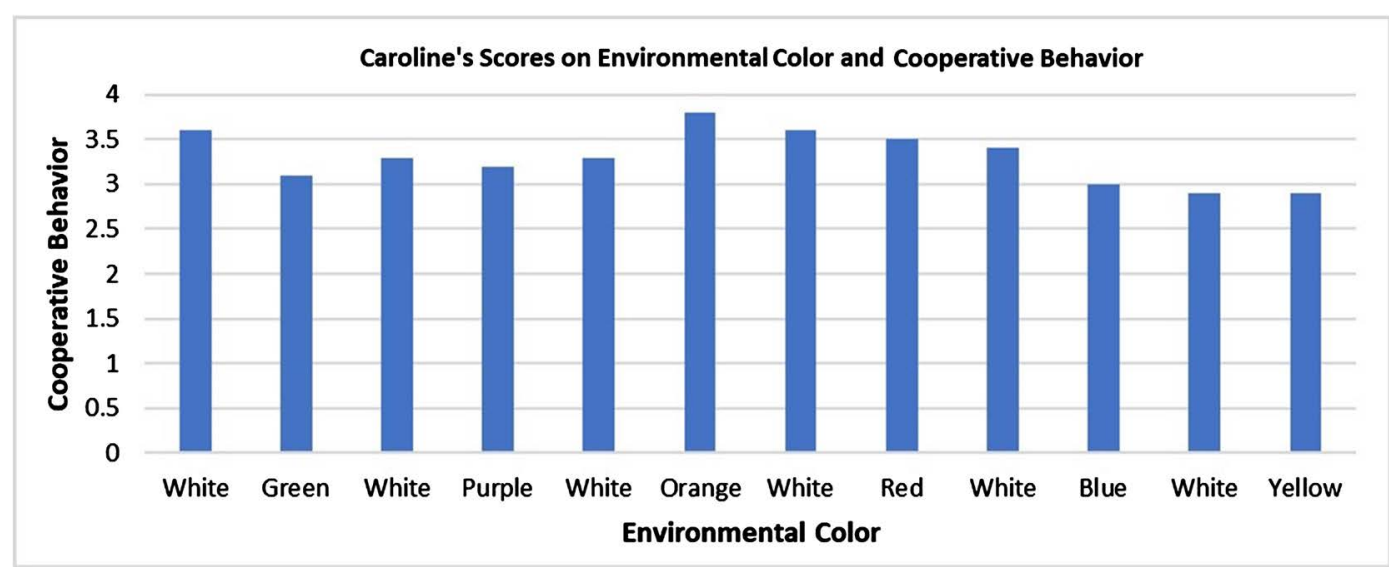

Figure 2. Caroline's scores on cooperative behavior and environmental color conditions. 


\section{Discussion}

This exploratory study used a single subject research design across 12 visible spectrum focal color and white conditions. The cooperative behavior of the young male participant appeared to be positively impacted by the focal wall colors while the cooperative behavior of the young female participant appeared to be negatively impacted by the focal wall colors, except for the orange condition. The primary limitation of this study is the small sample size. Generalizability is impossible with a single subject research design of two subjects. Fabric was used for all conditions. The fabric would influence the acoustics in the space by absorbing sound waves. Therefore, the play area would be quieter than a play area that used paint on the wall. Noise control is an important factor for children's well-being in a busy preschool environment; therefore, reduction of sound waves is advantageous for a calm environment.

This study is a first step in the study of wall color and cooperative behavior for children with sensory processing challenges. The findings add to the literature on the impact of environmental factors on children's behavior. Clearly the environment has significant meaning for children. Researchers are beginning to understand environmental factors in design which may impact children in the preschool environment.

Occupational therapists and preschool teachers may wish to incorporate more visible spectrum focal color on the lower section of walls in treatment rooms and preschool classrooms. They would act as accent walls that may act as a modifier for young children's attention in the environment. Modulating visual and auditory input are important goals for designing children's environments. This should be considered in future studies on wall color. Further study in a variety of educational settings would be warranted to investigate the impact of color on human behavior.

\section{Acknowledgements}

Thank you to Graduate Research Assistant, Adam Devitt; Sara Bartlett and Shyanne Cunningham; Cindy Bond; and to the College of Health and Human Sciences, Oregon State University for funding this research project.

\section{Conflicts of Interest}

The author declares no conflicts of interest regarding the publication of this paper.

\section{References}

Banaschewski, T., Ruppert, S., Tannock, R., Albrecht, B., Becker, A., Yebel, H., Seargeant, J. A., \& Rothenberger, A. (2006). Colour Perception in ADHD. Journal of Child Psychology and Psychiatry, 47, 568-572. https://doi.org/10.1111/j.1469-7610.2005.01540.x

Barrett, P., Davies, F., Zhang, Y., \& Barrett, L. (2015). The Impact of Classroom Design on Pupils' Learning: Final Results of a Holistic, Multi-Level Analysis. Building and En- 
vironment, 89, 118-133. https://doi.org/10.1016/j.buildenv.2015.02.013

Bornstein, M. H. (2007). Hue Categorization and Color Naming: Cognition to Language to Culture. In R. E. MacLaury, G. V. Paramei, \& D. Dedrick, (Eds.), Anthropology of Color (pp. 3-27). Amsterdam: John Benjamins. https://doi.org/10.1075/z.137.04bor

Bornstein, M. H. (2016). Emergence and Early Development of Color Vision and Color Perception. In A. J. Eliot, M. D. Fairchild, \& A. Franklin (Eds.), Handbook of Color Psychology. Cambridge: Cambridge University Press.

Dijkstra, K., Pieterse, M. E., \& Pruyn, A. T. H. (2008). Individual Differences in Reactions towards Color in Simulated Healthcare Environments: The Role of Stimulus Screening Ability. Journal of Environmental Psychology, 28, 268-277.

https://doi.org/10.1016/j.jenvp.2008.02.007

Dunn, W. (2006). Sensory Profile School Companion. London: Pearson.

Eliot, A. J. (2015). Color and Psychological Functioning: A Review of Theoretical and Empirical Work. Frontiers in Psychology, 6, 368.

https://doi.org/10.3389/fpsyg.2015.00368

Farran, E. K., Courbois, Y., Herwegen, J. V., Cruickshank, A. G., \& Blades, M. (2012). Colour as an Environmental Cue When Learning a Route in a Virtual Environment: Typical and a Typical Development. Research in Developmental Disabilities, 33, 900-908. https://doi.org/10.1016/j.ridd.2011.11.017

Gibson, E. J. (2000). Perceptual Learning in Development: Some Basic Concepts. Ecological Psychology, 12, 295-302. https://doi.org/10.1207/S15326969ECO1204_04

Gibson, J. J. (1979). The Ecological Approach to Visual Perception. Boston: MA: Houghton Mifflin.

Hidayetoglu, M. L., Yildirim, K., \& Akalin, A. (2012). The Effects of Color and Light on Indoor Way Finding and the Evaluation of the Perceived Environment. Journal of Environmental Psychology, 32, 50-58. https://doi.org/10.1016/j.jenvp.2011.09.001

Ichikawa, H., Hukami, K., Tanabe, S., \& Kawakami, G. (1978). Standard Pseudoisochromatic Plates: Part 1 for Congenital Color Vision Defects. Tokyo, Japan: Igaku Shoin.

Imhof, M. (2004). Effects of Color Stimulation on Handwriting Performance of Children with ADHD without and with Additional Learning Disabilities. European Child and Adolescent Psychiatry, 13, 191-198. https://doi.org/10.1007/s00787-004-0371-5

Iovino, I., Fletcher, J. M., Breitmeyer, B. G., \& Foorman, B. R. (1998). Colored Overlays for Visual Perceptual Deficits in Children with Reading Disability and Attention Deficit/Hyperactivity Disorder: Are They Differentially Effective? Journal of Clinical and Experimental Neuropsychology, 20, 791-806. https://doi.org/10.1076/jcen.20.6.791.1113

Jalil, N. A., Yunus, R. M., \& Said, N. S. (2012). Environmental Colour Impact upon Human Behavior: A Review. Procedia-Social and Behavioral Sciences, 35, 54-62. https://doi.org/10.1016/j.sbspro.2012.02.062

Jasmin, E., Gauthier, A., Julien, M., \& Hui, C. (2018). Occupational Therapy in Preschools: A Synthesis of Current Knowledge. Early Childhood Education Journal, 46, 73-82. https://doi.org/10.1007/s10643-017-0840-3

Joh, A. S., \& Spivey, L. A. (2012). Colorful Success: Preschoolers' Use of Perceptual Color Cues to Solve a Spatial Reasoning Problem. Journal of Experimental Child Psychology, 113, 523-534. https://doi.org/10.1016/j.jecp.2012.06.012

Kranowitz, C. (2003). The Out-of-Sync Child Has Fun: Activities for Kids with Sensory Integration Dysfunction. New York: Perigee.

Kyttä, M. (2004). The Extent of Children's Independent Mobility and the Number of Ac- 
tualized Affordances as Criteria for Child-Friendly Environments. Journal of Environmental Psychology, 24, 175-198. https://doi.org/10.1016/S0272-4944(03)00073-2

Lawson, L. M., \& Dunn, W. (2008). Children's Sensory Processing Patterns and Plan Preferences. Annual in Therapeutic Recreation, 16, 1-14.

Paulson, F. L. (1972). The Oregon Preschool Test of Interpersonal Cooperation.

Pitchford, N. J., \& Mullen, K. T. (2005). The Role of Perception, Language, and Preference in the Developmental Acquisition of Basic Color Terms. Journal of Experimental Child Psychology, 90, 273-302. https://doi.org/10.1016/j.jecp.2004.12.005

Pitchford, N. J., Davis, E. E., \& Scerif, G. (2009). Does Colour Preference Have a Role in Colour Term Acquisition? British Journal of Developmental Psychology, 27, 993-1012. https://doi.org/10.1348/026151008X399916

Read, M. A., Sugawara, A. I., \& Brandt, J. A. (1999). Impact of Space and Color in the Physical Environment on Preschool Children's Cooperative Behavior. Environment and Behavior, 31, 413-428. https://doi.org/10.1177/00139169921972173

Zhan, S., \& Ottenbacher, K. J. (2001). Single Subject Research Designs for Disability Research. Disability and Rehabilitation, 23, 1-8.

https://doi.org/10.1080/09638280150211202 